\title{
Communication framework to support more effective onsite construction monitoring
}

\author{
R. Y. Shrahily, B. Medjdoub, M. Kashyap \& M. L. Chalal \\ The Creative and Virtual Technologies Research Lab, \\ Nottingham Trent University, UK
}

\begin{abstract}
The UK construction industry has recently witnessed an increasing demand for cost-reduction strategies due to the strict government regulations on BIM implementation. This adoption will certainly lead to a continuous work improvement, better project delivery and communication. Although the UK government has set a target of 15-20\% saving on the costs of capital projects by the full implementation of BIM level 2 in 2016, this figure is unlikely to be met since the majority of construction companies are still spending approximately $£ 20$ billion per year on rebuilding and repairing the construction defects caused by miscommunication. This research addresses the problem of communication using traditional methods (i.e. communication through paper-based documents and drawings) and its impact during the construction phase in relation to clash detection. Next, we will present a communication framework using advanced visualisation technique such as augmented reality (AR) combined with a BIM model with an easy access to the IFC file on site for a compliance checking between the BIM model and the actual construction site. Subsequently, site inspection can be performed more efficiently, and with more reliability. Furthermore, early warning on future occurring clashes can be given. To reach our objectives, the research has been designed using real case scenario, following two phases of implementation. The first phase include the communication study and consists of determining users requiring assistance with regard to site monitoring and inspection, whereas the second, built on the results of the first phase to specify and implement the mobile AR system.

Keywords: building information modelling, augmented reality, communication studies, IFC file, tracking sensors, work breakdown structure, organization breakdown structure, construction responsibility assignment matrix.
\end{abstract}




\section{Introduction}

Due to the complex and diverse nature of construction industry, which is described as one of the vital sector in the UK, an approximate of $£ 20$ billion per year is being spent to rebuild and fix construction defects [1]. This is mainly caused by communication and collaboration failure between stakeholders. According to Project Communication Handbook [2], communication in this context simply means the information exchange focusing on creating an understanding environment between the groups working on construction site. This can be done by various ways such as exchanging calls, 2D drawings, text-based documents, onsite meetings, etc. It is generally believed that successful projects, usually have a high level of communication standards contributing to the overall project. Overall, lack of communication can be summarised as follows [1]:

1) Missing details in $2 \mathrm{D}$ execution drawings, where owners, contractors, designers and others share the responsibility to highlight errors that may lead to extra costs;

2) The extra time workers need to unexpectedly spend on site to understand unclear drawings;

3) Finally, the unavailability of critical information that is essential to complete the building task [1].

To reduce costs in the construction industry, UK government is committed to implement the BIM level 2 target by 2016. Thus, construction industry is required to keep pace with this objective. The reason behind this huge effort according to McNell et al. [3] is that BIM has reduced the overall construction costs dramatically and Giel et al. [4] estimated this saving by 15-40\% since 2006. However, to meet this goal, the main causes of construction defects need to be identified. For those reasons, the undertaken research aims to address a prominent aspect related to the construction defects and communication issues which is based on identifying the widely employed communication methods. Furthermore, defining the nature and frequency of construction defects occurring at different construction phase.

\section{Methodology}

The focus of this research is to develop a communication framework for detecting construction defects on site using BIM enhanced with mobile visualisation system using AR.

Qualitative methodology with actions research principles was chosen because the essence of this study is not only to understand the needs and obstacles facing different construction groups but also improve their working conditions Koshy [5]. A minimum of two iterations is adopted, starting with a general communication studies, followed by observation undertaken on construction site. This will be vital to identify potential AR system users. Afterwards, workshops will be held to allow participants from different backgrounds to assess and suggest improvements to the developed communication framework. Finally, these outcomes in addition to participants' comments will be utilised to improve the framework. 


\section{Site communication methods studies}

To develop the knowledge of communication process, the Work Breakdown Structure (WBS) and Organization Breakdown Structure (OBS) are used in this research to represent the construction processes as well as communication systems. WBS objective is to demonstrate how the project components are related and help to schedule different processes [6]; whereas, the purpose of OBS is to help assign duties or responsibilities to different working groups in relation to the scheduled processes. The outcomes resulting from the WBS and the OBS is used as input to define our suggested Responsibility Assignment Matrix (RAM) framework in order to represent communication tasks across the construction life-cycle. This framework will be discussed in depth in the following sections.

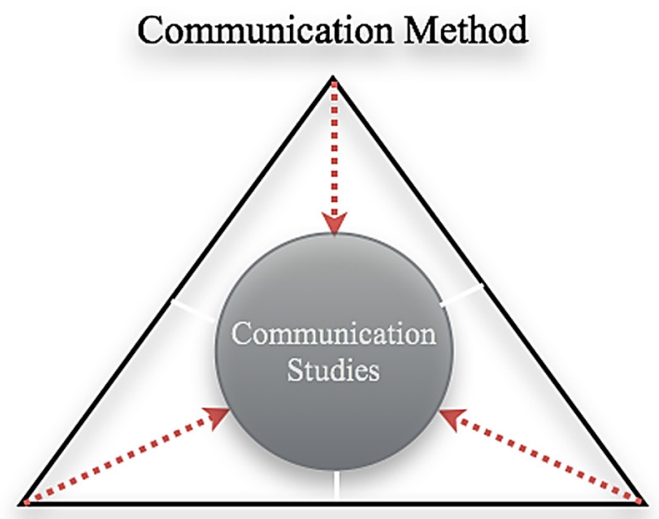

Construction Phase and Tasks

Project Team Work

Figure 1: Communication studies diagram.

\subsection{Work Breakdown Structure (WBS)}

WBS illustrates the necessary construction activities required for delivering the overall project [6]. Its aims to simplify as well as to facilitate the planning of different interrelated construction phases.

Based on a thorough investigation of literature review on construction processes in the [7], we have identified ten construction phases in most construction projects namely; site Preparations, Foundation work, Structure, Building Envelop, Interior Construction, Doors/Windows Installation, Electrical Installation, HVAC system, Building Services Installation and Finishing and decoration, respectively. 
198 Building Information Modelling (BIM) in Design, Construction and Operations

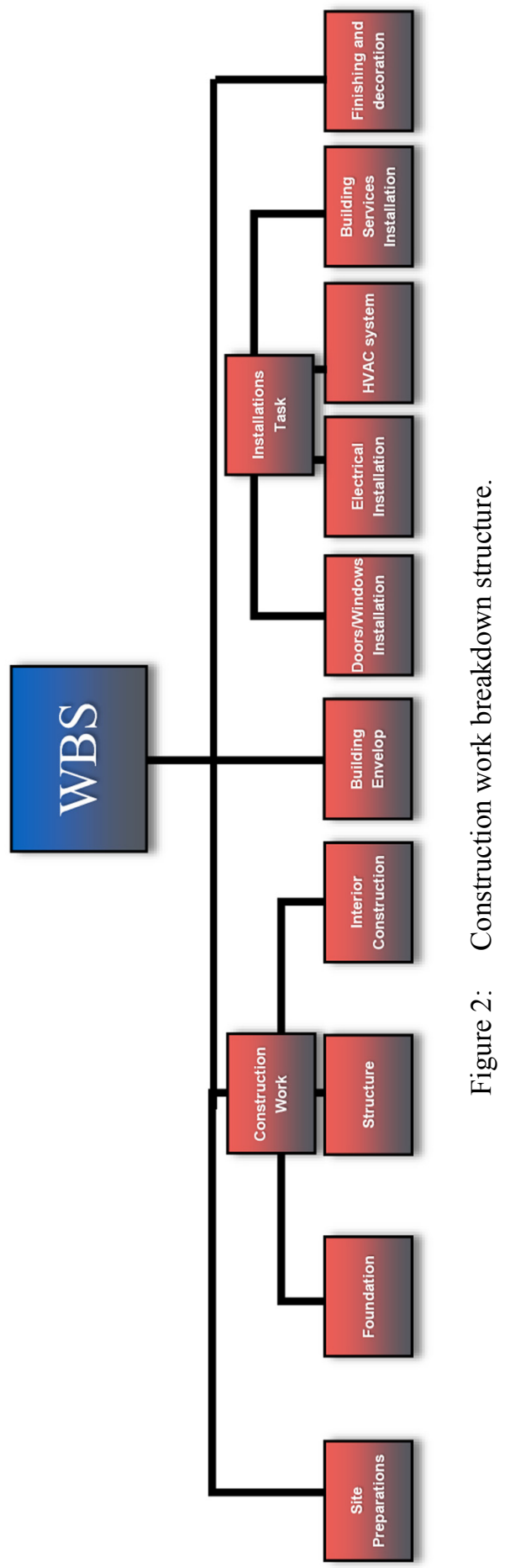




\subsection{Organization Breakdown Structure (OBS)}

The OBS is to describe the project team structure that groups personnel into small units. Each one is connected to a single manger or supervisor. Units are, in turn, subdivided into branches, which can be further subdivided again into sections with different responsibilities in large projects [8]. This framework will provide an adequate understanding of the group classifications and work responsibilities figure 3 .

The interest of this research is the teams operating on construction site directly which are as follows; Client/Owner, Design Team, Structural Design Team, HVAC Team, Task Manager/Foreman, Contractor, Site Manager, Workers and Suppliers.

\section{CRAM: construction responsibility assignment matrix}

Notice; research used a modified framework of RAM to meet the construction communication study's needs. In this study, the RAM is used to assign communication methods for inspection role to the team work operating onsite. In this contact it was called Construction Responsibility Assignment Matrix.

As described at an earlier stage, the RAM concept is an overlap between the WBS and the OBS outcomes [9]. The information resulting from the RAM will produce the raw material of the developed communication framework. To develop our framework we have adopted three communication methods namely; conventional drawings, onsite meetings, and text-based documents as indicated in figure 4. Based on numbers use against the construction phases, it was concluded that conventional drawings are the most used communication medium during the construction phase. Onsite meetings came second during 5 different stages namely Site preparation, foundation, structure, interior construction and building envelop. The text document communication method is mainly adopted by suppliers and contractors on site during 3 phases namely; windows/doors installations, electrical installations, HVAC system for material inspection and in the phase of finishing and decoration (see figure 5).

\section{Conclusion}

In this project we have suggested a new construction responsibility assignment matrix to identify the relationship between the construction phases, the stakeholder and the communication medium. This framework will be augmented with our next research stage aiming at identifying the defects occurring onsite versus the construction phases. This will help us to implement our AR visualization system targeting the appropriate construction phase and the stakeholder involved. Therefore, we will be able to augment the construction site with the next process and to check the compliance of the site with the BIM model. 


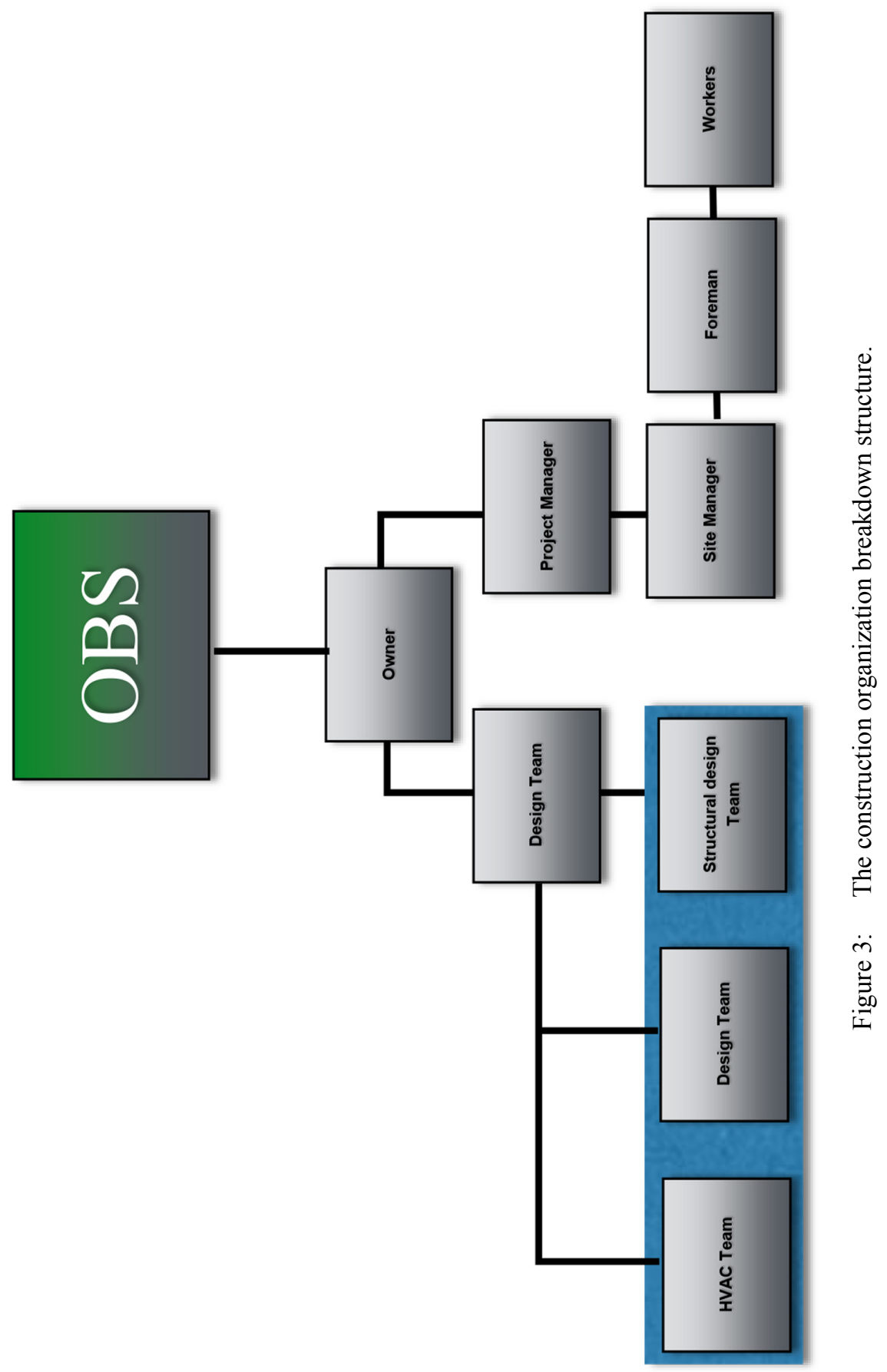




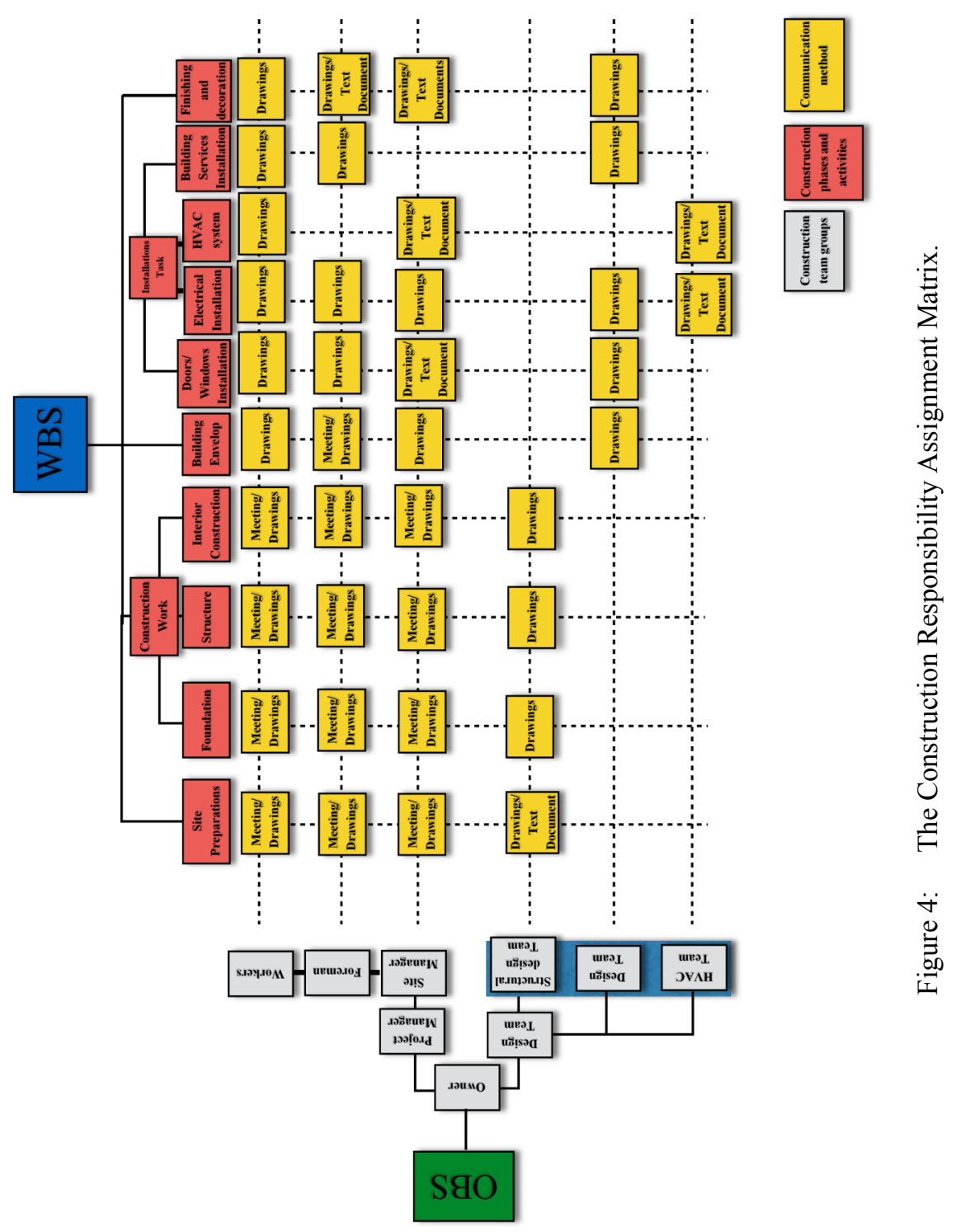




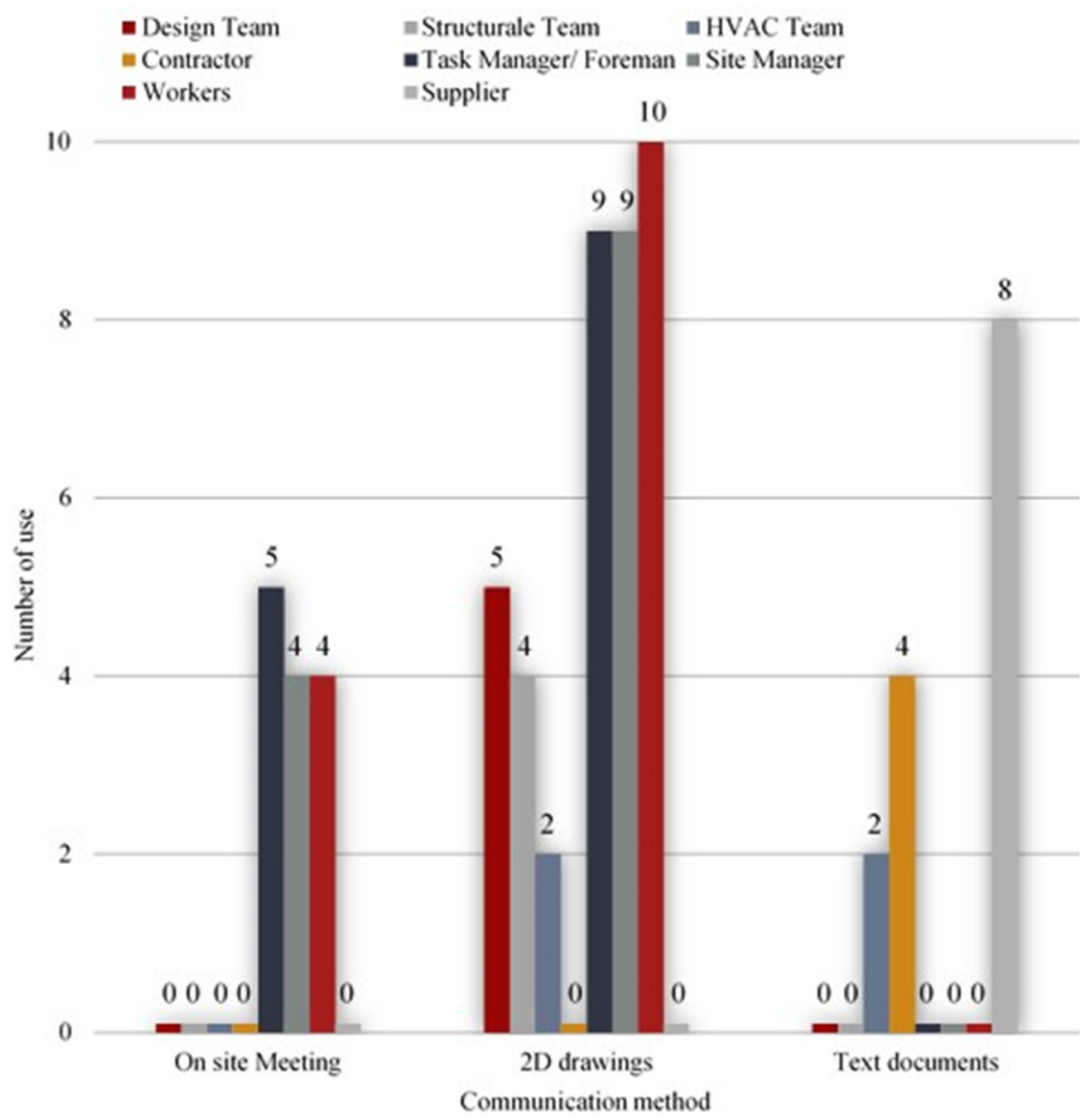

Figure 5: Showing the number of communication methods used to support on site construction inspection.

\section{References}

[1] Charles, J. BRE guidance on construction site communication, 2004.

[2] Office of Project Management Process Improvement, Project Communication Handbook, 2007.

[3] McNell, D. \& Allison, H. \& Black, W. \& Cukrow, M. \& Harrison, K. \& Sherred, T \& Shirley, M. \& Singh, R. \& Wilts, D. BIM. infoComm, International, 2008.

[4] Giel, B. \& R, Issa \& Olbina, S. Return on investment analysis of building information modeling in construction, Conference paper.

[5] Koshy, V. Action Research for Improving Practice A Practical Guide, 2005.

[6] Rajani, T. \& Shobha, V. Work Breakdown Structure of the Project, International Journal of Engineering Research and Applications, 2012. 
[7] Abdul Kadir, M. Construction Planning and Schedulin: A case Study of (Cadangan Membing Dan Menyiapkan Kulliyyah Untuk Universiti Antarabangsa Malaysia), 2006.

[8] Korneva, D. Description of the Turnkey Construction Project System for International and Russian Customers, 2012.

[9] Nevison, J. The Responsibility Assignment Matrix (RAM) Assigning Responsibilities for Deliverables to Roles, 2013. 